\title{
An RPC-PET prototype with high spatial resolution
}

\author{
A. Blanco ${ }^{\mathrm{a}, *}$, N. Carolino ${ }^{\mathrm{a}}$, C.M.B.A. Correia ${ }^{\mathrm{b}}$, R. Ferreira Marques ${ }^{\mathrm{a}, \mathrm{c}}$, \\ P. Fonte ${ }^{\mathrm{a}, \mathrm{d}}$, D. González-Díaz ${ }^{\mathrm{e}}$, A. Lindote ${ }^{\mathrm{a}}$, M.I. Lopes ${ }^{\mathrm{a}, \mathrm{c}}$, \\ M.P. Macedo ${ }^{\mathrm{b}, \mathrm{d}}$, A. Policarpo ${ }^{\mathrm{a}, \mathrm{c}}$ \\ ${ }^{a}$ LIP, Laboratório de Instrumentação e Fisica Experimental de Partículas, Dep-Física Univ. Coimbra, \\ Coimbra 3004-516, Portugal \\ ${ }^{\mathrm{b}}$ CEI, Centro de Electrónica e Instrumentação, Univ. Coimbra, Coimbra, Portugal \\ ${ }^{\mathrm{c}}$ Departamento de Física, Univ. Coimbra, Coimbra, Portugal \\ ${ }^{\mathrm{d}}$ ISEC, Instituto Superior de Engenharia de Coimbra, Coimbra, Portugal \\ ${ }^{\mathrm{e}}$ GENP, Grupo Experimental de Núcleos y Particulas, Fac. Física Univ. Santiago de Compostela, Santiago de Compostela, Spain
}

Available online 21 July 2004

\begin{abstract}
A small positron emission tomography system, based on the timing RPC technology has been built and tested. This first prototype is aimed at validating the expectations, derived from simulations, of a very high spatial resolution, which could be of value for the imaging of small animals. The system is composed of two counting heads, able to measure the photon interaction point in two dimensions, the transaxial dimension and the Depth of Interaction. Each head is composed of 16 independent stacked RPCs made from copper and glass (anode) electrodes. Point-like ${ }^{22} \mathrm{Na}$ sources were so far reconstructed, using the standard filtered back-projection algorithm, with a spatial accuracy of $0.6 \mathrm{~mm}$ FWHM, free of parallax error.
\end{abstract}

(C) 2004 Elsevier B.V. All rights reserved.

PACS: 29.40.Cs; 87.59.Vb; 87.62. + n

Keywords: Gaseous detectors; Photodetectors; Resistive plate chambers (RPC); Positron emission tomography (PET); Small animal imaging

\section{Introduction}

Positron Emission Tomography (PET) is a radiotracer imaging technique, in which tracer

\footnotetext{
*Corresponding author. Tel.: +351-29833465; fax: + 351239822358.

E-mail address: alberto@lipc.fis.uc.pt (A. Blanco).
}

compounds labelled with positron emitting radionuclides are injected into the object of study. These tracer compounds can then be used to track biomedical and physiological processes. Due to the high sensitivity when compared with other techniques, PET is a powerful tool for in vivo investigation of many biological process, which involve sub-picomolar concentrations of the 
affected molecules [1]. A representative example is the abnormal glucose metabolization, a signature of the presence of tumour cells.

One of the PET applications is on small animal tomography, applied in the development of new drugs, human disease studies and validation of gene therapies. In this modality, small animals, like transgenic mice and rats, are used as experimental models owing to its genetic likelihood with humans, short reproductive cycle and simple breeding. However, due to the small dimensions of these animals, dedicated high spatial resolution instruments are required, since it is difficult for existing animal PET scanners based on scintillators $[2,3]$ to achieve sub-millimetre spatial resolution.

The present work aims at validating the expectations, derived from simulations [4], of a system based on RPCs with sub-millimetre spatial resolution and free of parallax error. In this approach, based on the converter-plate principle [5], the detection of the incident $511 \mathrm{keV}$ photons, arising from the positron annihilation, is carried out through its conversion in an electron, inside a plate, and subsequent detection of the emitted electron. The material, the number of plates and the structure play an important role in the overall sensitivity of the system, but in this first prototype these parameters have not been yet optimised.

\section{Experimental set-up}

The system is composed of two counting heads, each one containing 16 stacked RPCs able to measure the photon interaction point in two dimensions: the transaxial dimension and the DOI. The third dimension, axial is not measured for the moment, but are easily included as it is shown in Ref. [6]. The extraordinary timing resolution of these detectors [7] has not been fully exploited in this first prototype.

\subsection{Detector}

Each head is built with 17 identical stacked plates, which define 16 independent sensitive gas gaps. The spacing is kept by $0.3 \mathrm{~mm}$ diameter

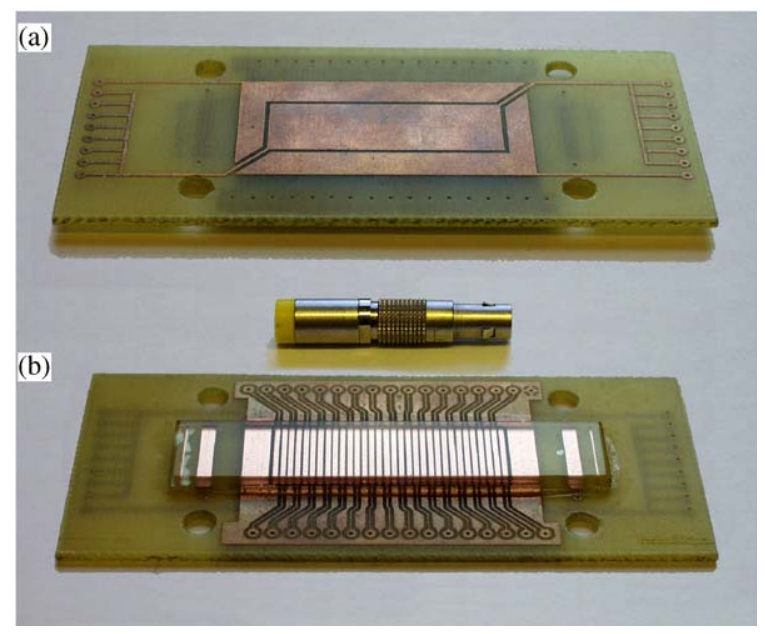

Fig. 1. Upper and lower view of one of the electrode plates. These are made from PCB and accommodate in one side (a) the metallic cathode of an RPC (PCB copper), and on the opposite side (b) the $2 \mathrm{~mm}$ thick glass anode of the next RPC and the 32 signal pickup strips, $1 \mathrm{~mm}$ wide, which sense the transaxial dimension.

nylon monofilaments. Fig. 1 shows one of these plates, made from standard printed circuit board (PCB), which accommodates in one side, Fig. 1(a), the metallic cathode of an RPC (PCB copper) and glued on the opposite side, Fig. 1(b), the $2 \mathrm{~mm}$ thick glass anode of the next RPC. High voltage is applied to the cathode. Under the glass anode 32 signal pickup strips, $1 \mathrm{~mm}$ wide, sense the transaxial dimension, covering an area of $32 \times 10 \mathrm{~mm}^{2}$.

The 17 plates are stacked on a common ceramic frame, which guarantees the precise alignment between the stacked electrodes and assures the necessary mechanical rigidity (Fig. 2). The stack is enclosed in a metallic gas-tight box, filled with the "standard" mixture: $\mathrm{C}_{2} \mathrm{H}_{2} \mathrm{~F}_{4} \quad 85 \%, \mathrm{SF}_{6} \quad 10 \%$, $\mathrm{C}_{4} \mathrm{H}_{10} 5 \%$.

\subsection{Readout electronics}

The charge induced in the cathodes, indicating the gas gap where the detection takes place (Depth of Interaction (DOI)), is individually read by 16 charge sensitive amplifiers, based on Analogue Devices OP467 chip. The charge induced in the strips, grouped together for each column, is read 


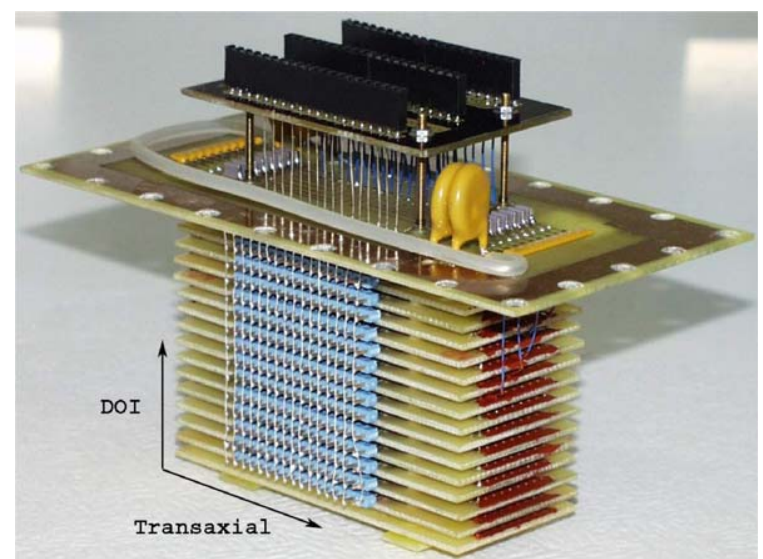

Fig. 2. Each of the counting heads, built of 16 stacked RPCs, is able to measure the photon interaction point in two dimensions, the transaxial dimension and the DOI.

by 32 similar amplifiers. The readout of the entire system comprises 96 charge sensitive channels.

All the analogical signals are sent to discriminator boards based on MAX912 chip, which indicate the firing gap inside each head and the digital pattern of the signals in the strips, determining the coarse transaxial position of the photon interaction point. The charge signals from the 32 strips in each head are summed in four groups, corresponding to strips separated by three and sent to a CAMAC-based charge integrating ADC (LeCroy 2249W). This information is used for interstrip position interpolation, allowing a precise determination of the transaxial dimension.

The control and acquisition of all the signals is commanded by a Pic16F877 microcontroller that transfers the data to the acquisition computer, via a FIFO interface board.

\section{Results}

\subsection{Experimental spatial resolution}

The two counting heads were placed as close as practically possible $(40 \mathrm{~mm})$ to improve the sensitivity of the system. Three point-like ${ }^{22} \mathrm{Na}$ positron sources, with a total activity of $15 \mu \mathrm{Ci}$, were enclosed in a plastic material and placed in the Field of View (FOV). Two of them, grouped together, were spaced by $1 \mathrm{~mm}$ and situated at the centre of the FOV, while the remaining one is separated $10 \mathrm{~mm}$ from the centre.

The three sources were imaged and reconstructed by the standard algorithm of filtered back-projection, without any manipulation for the image enhanced, yielding the images shown in Fig. 3(a).

Due to the limitations of this prototype, incomplete ring and no object rotation, only angles between $\pm 40^{\circ}$ are available, some extrapolation being necessary to obtain the remaining angles for the image reconstruction. Due to the punctual nature of the sources, the data could be extrapolated for the remaining angles using the well-known sinogram curve, without any image corruption.

(a)
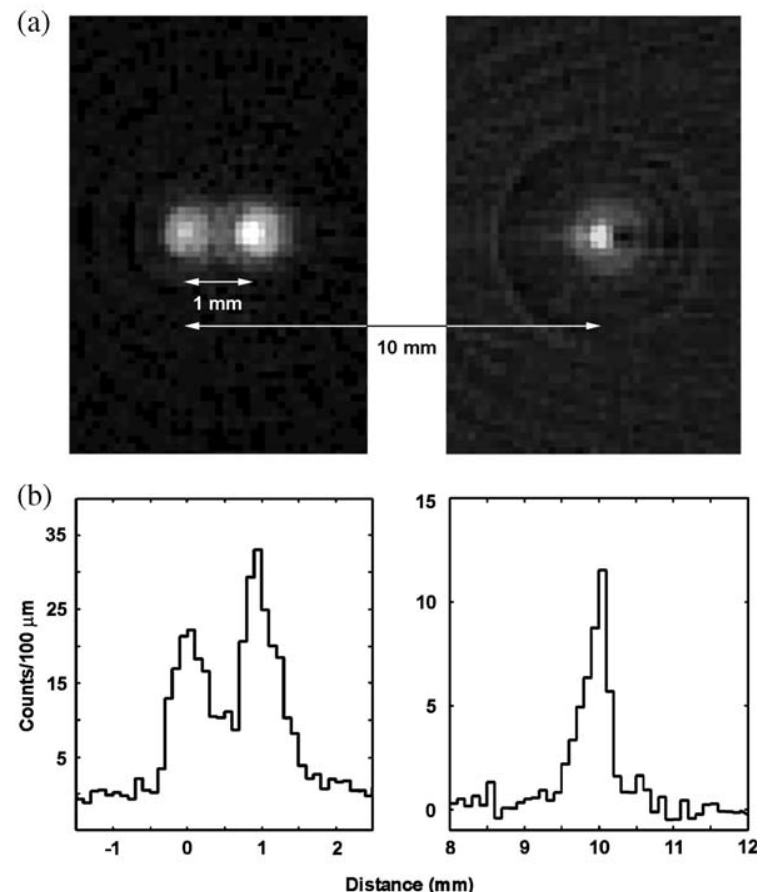

Fig. 3. (a) Image reconstruction, using the standard algorithm of filtered back-projection (no image enhancement), of three point- like ${ }^{22} \mathrm{Na}$ positron sources surrounded by plastic and seen by the two counting heads. (b) Point Spread Function of the sources showing a width of $0.6 \mathrm{~mm}$ FWHM. 
Table 1

Comparison between different small animal PET parameters and the expected parameters of the RPC-PET

\begin{tabular}{|c|c|c|c|c|}
\hline & $\begin{array}{l}\text { Quad HIDAC } \\
\text { (32 modules) [8] }\end{array}$ & YAP-PET [2] & $\begin{array}{l}\text { MicroPET }^{\circledR} \\
\text { II [3] }\end{array}$ & RPC-PET ${ }^{\mathrm{a}}$ \\
\hline Central point absolute sensitivity (cps/kBq) & $18^{\mathrm{b}}$ & $17.3(\varnothing=150 \mathrm{~mm})^{\mathrm{c}}$ & $22.6^{\mathrm{d}}$ & $9^{e}$ \\
\hline Image spatial resolution (mm) FWHM & $1 \mathrm{~mm}$ (uniform) & $<1.8$ (uniform) & 1.07 & $\leqslant 0.6$ (uniform) \\
\hline Time resolution (ns) FWHM & - & 2 & 3 & $<300 \mathrm{ps}$ \\
\hline Window time (ns) & $<80$ & $<5$ & $<10$ & $<1$ \\
\hline FOV (mm) & $\begin{array}{l}170 \varnothing \times 280 \\
\text { (axial) }\end{array}$ & $40 \times 40 \times 40$ & $\begin{array}{l}160 \varnothing \times 49 \\
\text { (axial) }\end{array}$ & $150 \varnothing \times 300$ (axial) \\
\hline
\end{tabular}

${ }^{\text {a }}$ Values calculated assuming an efficiency of $10 \%$ (a conservative value from the simulation) and a relative solid angle of $85 \%$.

${ }^{\mathrm{b}}$ Scatter corrected, some intrinsic energy threshold.

${ }^{\mathrm{c}} \mathrm{Scatter}$ corrected, $50 \mathrm{keV}$ energy threshold.

${ }^{\mathrm{d}}$ No scatter corrected, 250-750 keV energy threshold.

${ }^{\mathrm{e}}$ No scatter corrected.

Fig. 3(a) shows the two central sources clearly resolved. The third source, separated $10 \mathrm{~mm}$ from the centre of the FOV, shows an unaltered resolution, confirming the parallax free capability of the system. The corresponding Point Spread Function was determined by reconstructing point sources and shows a width of $0.6 \mathrm{~mm}$ FWHM (Fig. 3(b)).

\subsection{Expected performance}

In Table 1, three different small animal PET tomographs are compared with the expected performance of a full-sized RPC-PET system. The central point absolute sensitivity is calculated assuming an efficiency per photon of $10 \%$ (a conservative value derived from the simulations) and a relative solid angle coverage of $85 \%$.

In spite of the relatively low efficiency, the central point absolute sensitivity reaches a value of $9 \mathrm{cps} / \mathrm{kBq}$, owing to the large spatial acceptance of the scanner.

The very good timing resolution of these detectors, $\sim 300$ ps FWHM for the time difference between photon pairs, allows to contemplate the use of a time window narrower than $1 \mathrm{~ns}$, reducing the random events rate and improving the noise equivalent count rate $(\mathrm{NECR})^{1} \_$a common figure

\footnotetext{
${ }^{1}$ The number of true coincidences that would create an image of similar quality in the absence of noise (scattered and random coincidences).
}

of merit for comparing tomograph performance. The necessary time acquisition for a given image quality is then significantly reduced [9]. The excellent spatial resolution, $0.6 \mathrm{~mm}$ FWHM over the entire FOV, will allow images of unprecedented high resolution to be taken.

\section{Conclusions}

We have presented first results of a small PET system based on the timing RPC technology.

The system is composed of two counting heads, each built from 16 stacked RPCs made from copper and glass electrodes.

An image position resolution of $0.6 \mathrm{~mm}$ FWHM, free of parallax error, was demonstrated for point-like ${ }^{22} \mathrm{Na}$ sources.

This technology seems to be very appropriate for small animal PET studies, providing a very high spatial resolution and medium sensitivity at a low cost.

\section{Acknowledgements}

The authors gratefully acknowledge the special collaboration of C. Gil, F. Marques, A. Pereira, N. Chichorro, and L. Fazendeiro.

This work was financed by Fundação para a Ciência e Tecnologia project POCTI/FNU/49513 / 2002. 


\section{References}

[1] A. Del Guerra, et al., Q. J. Nucl. Med. 46 (2002) 35.

[2] A. Del Guerra, et al., IEEE Trans. Nucl. Sci. NS 45 (6) (1998) 3105 http://www.ise-srl.com/YAPPET/Yap-doc.htm.

[3] Yuan-Chuan Tai, et al., Phys. Med. Biol. 48 (2003) 1519.

[4] P. Fonte, et al., Nucl. Instr. and Meth. A 508 (2003) 88.
[5] J.E. Bateman, et al., Nucl. Instr. and Meth. A 225 (1984) 209.

[6] P. Fonte, et al., Nucl. Instr. and Meth. A 508 (2003) 70.

[7] P. Fonte, et al., Nucl. Instr. and Meth. A 443 (2000) 201.

[8] http://www.oxpos.co.uk.

[9] W.W. Moses, IEEE Trans. on Nucl. Sci.NS 50 (5) (2003) 1325. 